\title{
Unitary equivalence of operators and dilations
}

\author{
by \\ Chafiq Benhida (Lille)
}

\begin{abstract}
Given two contractions $T$ and $T^{\prime}$ such that $T^{\prime}-T$ is an operator of finite rank, we prove, under some conditions, the unitary equivalence of the unitary parts of the minimal isometric dilations (respectively minimal co-isometric extensions) of $T$ and $T^{\prime}$.

1. Introduction. Let $\mathcal{H}$ be a separable Hilbert space. $\mathcal{L}(\mathcal{H})$ will denote the algebra of all bounded operators on $\mathcal{H}$. An operator $T \in \mathcal{L}(\mathcal{H})$ is a contraction if $\|T\| \leq 1$ or equivalently $I-T^{*} T$ is positive. Note also that any contraction $T$ has a canonical decomposition $T=T_{1} \oplus T_{2}$ into a completely nonunitary contraction $T_{1}$ and a unitary operator $T_{2}$. We say that $T$ is absolutely continuous if the unitary part $T_{2}$ is absolutely continuous, which means that the spectral measure of $T_{2}$ is absolutely continuous with respect to the Lebesgue measure.

The defect operators are the positive operators defined by $D_{T}=$ $\left(I-T^{*} T\right)^{1 / 2}, D_{T^{*}}=\left(I-T T^{*}\right)^{1 / 2}$; the defect spaces are $\mathcal{D}_{T}=\overline{D_{T} \mathcal{H}}$ and $\mathcal{D}_{T^{*}}=\overline{D_{T^{*}} \mathcal{H}}$.
\end{abstract}

2. Main result. Recall the following result which is due to Carey ([2]).

Lemma 2.1. Let $W_{1}, W_{2}$ be isometries on a Hilbert space such that $W_{1}-W_{2}$ has finite rank. If $W_{i}=W_{i}^{u} \oplus W_{i}^{p}$ is the Wold decomposition of $W_{i}(i=1,2)$, then the absolutely continuous parts of $W_{1}^{u}$ and $W_{2}^{u}$ are unitarily equivalent.

We shall use this result to prove the following one.

Proposition 2.2. Let $T_{1}, T_{2} \in \mathcal{L}(\mathcal{H})$ be contractions such that $T_{2}-T_{1}$ is a finite rank operator and the dimension of $\bigvee_{k>0}\left(D_{T_{i}}^{2}\right)^{k}\left(T_{1}^{*} T_{1}-T_{2}^{*} T_{2}\right) \mathcal{H}$ is finite for $i=1$ or $i=2$. If $W_{T_{i}}$ is the minimal isometric dilation of $T_{i}$ for $i=1,2$, then the absolutely continuous parts of $W_{T_{1}}^{u}$ and $W_{T_{2}}^{u}$ are unitarily equivalent.

To prove the last proposition, we need the following lemma:

2000 Mathematics Subject Classification: 47A20, 47A55. 
Lemma 2.3. Let $A, B$ be positive operators on a Hilbert space $\mathcal{H}$ such that $A-B=R$ is a finite rank operator. If the dimension of $\bigvee_{k \geq 0} B^{k} R \mathcal{H}$ $=\mathcal{M}$ is finite, then $\sqrt{A}-\sqrt{B}$ is a finite rank operator.

Proof. Consider a family of polynomials $q_{n}$ that is convergent to the square root function uniformly on $\sigma(A) \cup \sigma(B)$. We then have $\sqrt{A}=$ $\lim _{n} q_{n}(A)$ and $\sqrt{B}=\lim _{n} q_{n}(B)$. Let $q_{n}=\sum_{m=0}^{M_{n}} a_{n, m} x^{m}$. Then

$$
\sqrt{A}-\sqrt{B}=\lim _{n}\left(q_{n}(A)-q_{n}(B)\right)=\lim _{n} \sum_{m=0}^{M_{n}} a_{n, m}\left(A^{m}-B^{m}\right) .
$$

It is quite clear that we have by induction

$$
A^{m}-B^{m}=R A^{m-1}+B R A^{m-2}+\cdots+B^{m-2} R A+B^{m-1} R .
$$

Under the assumptions made on $A$ and $B$, the range $A^{m}-B^{m}$ has a finite dimension since it is included in $\mathcal{M}$. This ends the proof.

REMARK. It is an interesting question whether the statement of the lemma given above remains true without the condition on $\mathcal{M}$. For example, consider $A=\sum_{n \geq 0} \alpha_{n}^{2} e_{n} \otimes e_{n}$ and $B=A+f \otimes f$, which is a rank one perturbation of $A$. Assume that $f=\sum_{n \geq 0} \beta_{n} e_{n}$. It is clear that $A$ and $B$ are positive operators. Could we choose the $\alpha_{n}$ and $\beta_{n}$ such that $\sqrt{A}-\sqrt{B}$ is not a finite rank operator?

Proof of Proposition 2.2. Set $\mathcal{D}=\mathcal{D}_{T_{1}} \vee \mathcal{D}_{T_{2}}$ and $\mathcal{K}=\mathcal{H} \oplus \mathcal{D} \oplus \mathcal{D} \oplus \cdots$. Consider on $\mathcal{K}$ the two isometries

$$
\widetilde{W}_{i}=\left(\begin{array}{cccc}
T_{i} & 0 & 0 & \cdots \\
D_{T_{i}} & 0 & 0 & \cdots \\
0 & I & 0 & \cdots \\
0 & 0 & I & \cdots \\
\vdots & \vdots & \vdots & \ddots
\end{array}\right)=\left(\begin{array}{cc}
T_{i} & 0 \\
D_{T_{i}} & S^{\alpha}
\end{array}\right), \quad i=1,2 .
$$

where $\alpha$ is the dimension of $\mathcal{D}$. It is easy to see, by the construction of the isometric dilation given in [3], that $\widetilde{W}_{i}$ is the direct sum of the minimal isometric dilation $T_{i}$ and an (eventual) shift. Then

$$
\widetilde{W}_{1}-\widetilde{W}_{2}=\left(\begin{array}{cc}
T_{1}-T_{2} & 0 \\
D_{T_{1}}-D_{T_{2}} & 0
\end{array}\right)
$$

By using Lemma 2.3 with $D_{T_{1}}^{2}$ and $D_{T_{2}}^{2}$ instead of $A$ and $B$, we see that $\widetilde{W}_{1}-\widetilde{W}_{2}$ is a finite rank operator and Carey's result gives the announced statement.

COROLlary 2.4. Let $T_{1}, T_{2} \in \mathcal{L}(\mathcal{H})$ be contractions such that $T_{2}-T_{1}$ is a finite rank operator. If $W_{T_{i}}$ is the minimal isometric dilation of $T_{i}$ for 
$i=1,2$, then the absolutely continuous parts of $W_{T_{1}}^{u}$ and $W_{T_{2}}^{u}$ are unitarily equivalent when one of the following statements is satisfied:

(i) $T_{1} \mid \mathcal{D}_{T_{1}}=0$,

(ii) $\left(D_{T_{1}}^{2}-D_{T_{2}}^{2}\right) \mathcal{H}$ is invariant under $D_{T_{1}}^{2}$ or $D_{T_{2}}^{2}$,

(iii) $D_{T_{1}}$ or $D_{T_{2}}$ is a finite rank operator.

Proof. (i) Let $T_{1} \mid \mathcal{D}_{T_{1}}=0$. In this special case $D_{T_{1}}=P_{\mathcal{D}_{T_{1}}}$ is the orthogonal projection on $\mathcal{D}_{T_{1}}$, and this obviously gives the assumption of Proposition 2.2 .

(ii) If $\left(D_{T_{1}}^{2}-D_{T_{2}}^{2}\right) \mathcal{H}$ is invariant under $D_{T_{1}}^{2}$ or $D_{T_{2}}^{2}$, then

$$
\bigvee_{k \geq 0}\left(D_{T_{i}}^{2}\right)^{k}\left(T_{1}^{*} T_{1}-T_{2}^{*} T_{2}\right) \mathcal{H}=\bigvee_{k \geq 0}\left(D_{T_{i}}^{2}\right)^{k}\left(D_{T_{1}}^{2}-D_{T_{2}}^{2}\right) \mathcal{H}
$$

has finite dimension for $i=1$ or $i=2$.

(iii) If $D_{T_{1}}$ or $D_{T_{2}}$ is a finite rank operator, then both are, since $D_{T_{1}}^{2}-D_{T_{2}}^{2}$ has finite rank.

Note that with (iii) we retrieve the result obtained in [1].

Of course there is a dual version of Proposition 2.2. This is due to the fact that the adjoint of the minimal isometric dilation of $T^{*}$ is actually the minimal co-isometric extension of $T$. The corresponding result can be formulated as follows.

Proposition 2.5. Let $T_{1}, T_{2} \in \mathcal{L}(\mathcal{H})$ be contractions such that $T_{2}-T_{1}$ is a finite rank operator and the dimension of $\bigvee_{k \geq 0}\left(D_{T_{i}^{*}}^{2}\right)^{k}\left(T_{1} T_{1}^{*}-T_{2} T_{2}^{*}\right) \mathcal{H}$ is finite for $i=1$ or $i=2$. If $B_{T_{i}}$ is the minimal co-isometric extension of $T_{i}$ for $i=1,2$, then the absolutely continuous parts of $B_{T_{1}}^{u}$ and $B_{T_{2}}^{u}$ are unitarily equivalent.

\section{References}

[1] C. Benhida and D. Timotin, Finite rank perturbations of contractions, Integral Equations Operator Theory 36 (2000), 253-268.

[2] R. W. Carey, Trace class perturbations of isometries and unitary dilations, Proc. Amer. Math. Soc. 45 (1974), 229-234.

[3] B. Sz.-Nagy and C. Foiaş, Harmonic Analysis of Operators on Hilbert Space, NorthHolland, Amsterdam, 1970.

UFR de Mathématiques, CNRS-UMR 8524, Bât. M2

Université des Sciences et Technologies de Lille

F-59655 Villeneuve d'Ascq Cedex, France

E-mail: benhida@math.univ-lille1.fr 